\title{
Derivation of various NONMEM estimation methods
}

\section{Yaning Wang}

\section{Erratum to: J Pharmacokinet Pharmacodyn (2007) 34:575-593} DOI 10.1007/s10928-007-9060-6

On page 586 , the term $2 f^{\prime}\left(\hat{\eta}_{i}, \theta\right) \sigma^{2} \omega^{2}$ in

$$
\begin{aligned}
“= & \text { constant }+\log \left[f\left(\hat{\eta}_{i}, \theta\right)^{2} \sigma^{2}+f^{\prime}\left(\hat{\eta}_{i}, \theta\right)^{2} \omega^{2}+2 f^{\prime}\left(\hat{\eta}_{i}, \theta\right) \sigma^{2} \omega^{2}\right] \\
& +\frac{\left[y_{i}-f\left(\hat{\eta}_{i}, \theta\right)\right]^{2}}{f\left(\hat{\eta}_{i}, \theta\right)^{2} \sigma^{2}}+\frac{\hat{\eta}_{i}^{2}}{\omega^{2}}
\end{aligned}
$$

should be changed to $2 f^{\prime}\left(\hat{\eta}_{i}, \theta\right)^{2} \sigma^{2} \omega^{2}$.

The online version of the original article can be found under doi:10.1007/s10928-007-9060-6.

\section{Y. Wang $(\varangle)$}

Food and Drug Administration, Office of Clinical Pharmacology, CDER,

WO21 RM3662 HFD-880, 10903 New Hampshire Avenue,

Silver Spring, MD 20993, USA

e-mail: yaning.wang@fda.hhs.gov 